\title{
Application of UDK Technology in Digital Display of Cultural Heritage Chen Jieping
}

Qing city digital science and technology institute, Beijing, 100085, china

\begin{abstract}
Keywords: UDK technology, unreal engine, virtual reality (VR), cultural heritage digital display
\end{abstract}
\begin{abstract}
With the development of virtual reality display technology of cultural heritage, the virtual reality display technology is developed more deeply and widely, the sense of reality and immersion put forward higher requirements, more and more people pay more attention to the attribute information of cultural relics. UDK technology not only has high fidelity 3D rendering technology, with the image quality optimization, adaptive partition layer for the image is obtained, and the real-time shadow rendering technology is taken to realize the cultural heritage manually tours and interactive roaming function automatically. It also has the open script programming interface, and it can connect the database through the dynamic link library, and link rich scene cultural background of each interface. This combination of basic information database and efficient virtual simulation technology can provide a visual and cultural feast for the user, and it has a great application prospect in the field of cultural heritage digital display.
\end{abstract}

\section{Introduction}

With the development of 3D graphics technology, virtual reality technology has been widely used in the field of city planning, games design, cultural relic protection. Combining with the multimedia technology and VR, the application of it shows so broad and important in the realization, such as remote monitor and control, videophone conference, and distance multimedia teaching, etc. even in the military war and training, the network and virtual reality technology also will plays an important role ${ }^{[1]}$. The combination of virtual reality technology and network technology will enhance the display and use of the protection of cultural heritage, the development is taken to a a new stage. For many cultural heritage addresses, there is no real and leaving only the site of cultural heritage, such as Old Summer Palace, we can restore the history through virtual reality technology in comprehensive, vivid, realistic conditions. When we are immersed in a virtual three-dimensional scene unlimited feeling in cultural heritage, at the same time, we want to get more information in the scene of cultural relics, such as cultural relics, material, background story, etc. here we call it "attribute information". Combined with the virtual scene simulation technology, the construction of multimedia applications, building the computer network platform, the realization of remote communication and control is obtained. At present, there is seldom software platform which can meet the high efficiency and high quality virtual display and view the properties ${ }^{[2]}$.

At present, the research of virtual scene attribute display ideas can be divided into two parts as:

On the one hand, with the GIS (Geographic Information System) technology application, more and more people require to deal with the problems in 3D space, two dimensional GIS data model and data organization are taken as the basis, 3D GIS is developed. Three dimensional GIS can storage and manage a lot of attribute information. Starting from the database to the 3D virtual technology, it has a strong property management functions, including query, modify ${ }^{[3-6]}$.

On the other hand, the 3D visualization and 3D space object management are coupled up, forming an integrated system. The 3D GIS technology has an obvious disadvantage, that a large number fine 3D model display rendering effect is not ideal, in all aspects such as the complexity of the model, material, physical properties are far less than the 3D modeling such as 3DMAX, Maya and others.

In the cultural heritage digital display process, the rendering of models in our cultural heritage digital display requirements are higher. Therefore, we chose second research directions, that choose a $3 \mathrm{D}$ visualization platform, the query techniques of attribute of 3D high precision model is taken, several software platforms are compared and shown in Figure 1. The cultural heritage digital 
display platform based on UDK can overcome the defects of three-dimensional GIS platform render software, and it can extend the data completeness of cultural heritage, promote information sharing, the cultural heritage exhibition is not only the visual presentation, but also cultural exhibition, it is one of the best tools for cultural heritage digital display.

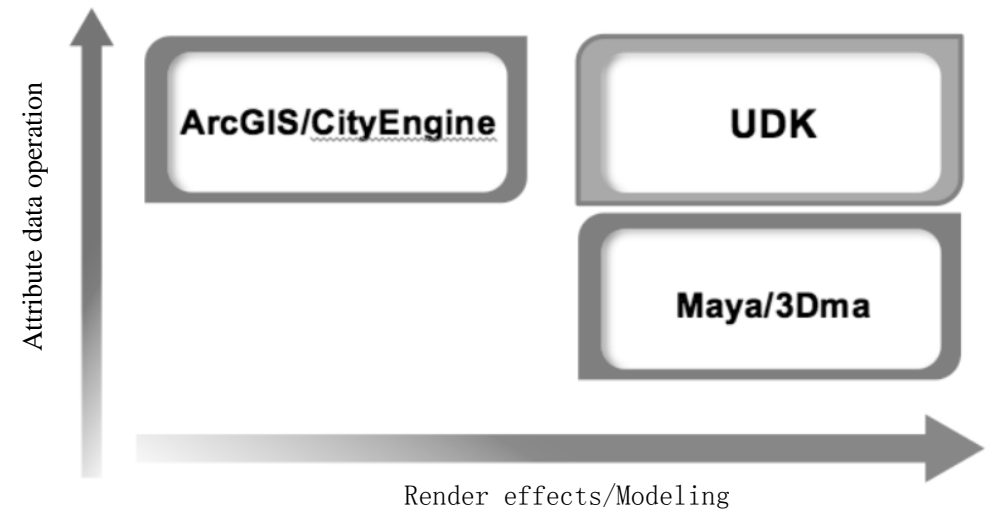

Figure. 1 Comparison of several kinds of 3D software platforms

\section{Introduction UDK Technology}

Unreal Development Kit is referred to as UDK, it is a set of strong and free game engine development kit, it can be used for the production of high-end 3D games or scene display. UDK uses the Unreal Engine 3 technology as driver, and it has a strong compatibility script Unreal Script. Unreal 3 is a set of DirectX 9/10 PC, Xbox 360, PlayStation 3 platform has he integral commercial game development framework, and it provides a large array of core technology, content editing tools, support for high-end development team based project construction. The Unreal3 engine is also integrated with physics engine, SpeedTree engine, sound engine, AI engine, and set the scene editor, animation editor, and other editors and so on, combined with the object-oriented UnrealScript language, and can provide many kinds of interactive experience.

Several advantages of UDK technologies are presented as:

(a)Ultra fine 3D rendering is completely;

(b) Multithreaded architecture makes the rendering efficiency higher;

(c) Completely with a strong Unreal Editor editing tool, set the scene modeling and animation etc.;

(d) Completely with Unreal Script scripting language, can be programmed to realize some special functions;

Unreal Editor

The editor is integrated with powerful scene editing, model editing, physical characteristics editing, animation editing and particle editing, multiple editors are combined together, it has the WYSIWYG features, greatly increased the system easier to use. The most commonly used tools in the editor are shown as: the content viewer, editor, Kismet event trigger, Matinee animation editor, particle editor, static mesh editor etc. With the editor, the game makers can zero programming to produce a fine 3D game. The diagram of the visual simulation frame based on UDK technology is shown in Figure 2 


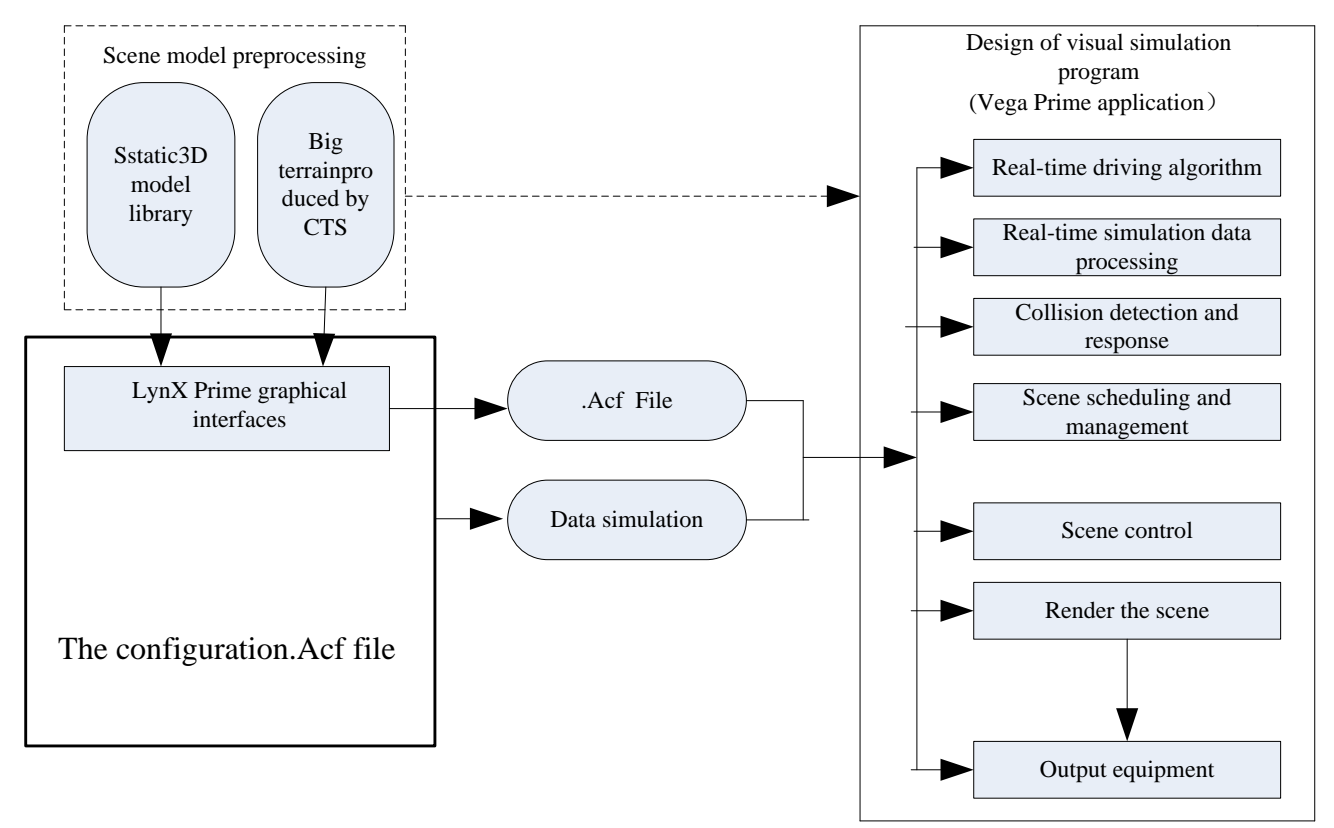

Figure 2. Diagram of the visual simulation frame based on UDK technology

Editor function is strong, but some of the expansion functions need the supplement functions with the UnrealScript scripting language. Create a new class by UnrealScript, the type of expansion provide the basis for the generic function in a custom class, in order to meet the needs of the game. Elements of the game cannot be achieved may need Editor, can be created in UnrealScript. UnrealScript provides automatic garbage collection mechanism. It has simple single inheritance mechanism, and it has type checking at compile time, its grammar and $\mathrm{C} / \mathrm{C}++/$ Java code have similarity. UnrealScript provides an open interface for a DLL dynamic library for programming, it provides a dynamic link library makes the game program be external, in order to achieve some functions that the UDK does not have itself, such as database connection etc.

\section{Design and implementation of display system}

\section{Data import}

Modeling software such as MAYA, 3DMAX and other popular software cannot provide us with interactive query control, but in the three-dimensional modeling advantages is obvious in no doubt, this study integrated the advantages of 3D software, using 3DMAX to design 3D model, using the UDK development environment as introduction, and then the corresponding interactive control is designed for rendering and reality implementation. This not only reduces the time and difficulty of modeling, but also to reduce the cost of the study UDK modeling project group, more importantly, it can improve the speed of system development. According to the different amount of data, we have different methods as follows:

(1) The model is less

In the less model condition, in the model, the case of small amount of data, we can directly export 3DX files for 3DMAX as .ase or. fbx format, then import the file format through the browser. If you have already made maps in 3DMAX's words, also need to be mapped into the system, and in the content browser settings link configuration.

(2) Model with large number of data

When the 3D model has the huge amount of data, the structure is complex, we can use two times transformation approach. 3DS format will be converted to binary, because UDK has provided an open read binary format, so you can read the binary file conversion, regenerated into UDK models. In addition to the introduction of UDK model format requirements strictly, single model cannot be more than 65535; mapping using.tga format, alpha mapping has the 24 channel, a channel for the 32; and all documents must be English path. 


\section{Database connection}

For example, building model has a lot of information, we compile this information as its attribute data, UDK itself does not have the attribute of the data storage function, but UnrealScript provides for developers to call external DLL (Dynamic Link Library) interface (DLLBind), so we can through this interface attribute database manage the module of DLL, and database communication, read, query, modify the contents of the database can be realized. Therefore, in this study, the information data attributes are related to the storage of SQLite data model in the external, UDK through the DLLBind call external C++ compiler DLL and attribute database interaction is obtained.

The DLLBind interface can transfer parameters and receive the return value. But when the return data are Chinese characters, is needs for transcoding form UTF-16 to GB2312. So if you need to modify the Chinese character in database, also need to go back to the UTF-16 GB2312, otherwise there will be garbled phenomenon.

The database operations DLL with $\mathrm{C}++$ compiled as: connect to the database, according to the conditions of data query, return the query results, insert data, delete data and other functions. In the database and scene model, the unique ID is used to connect.

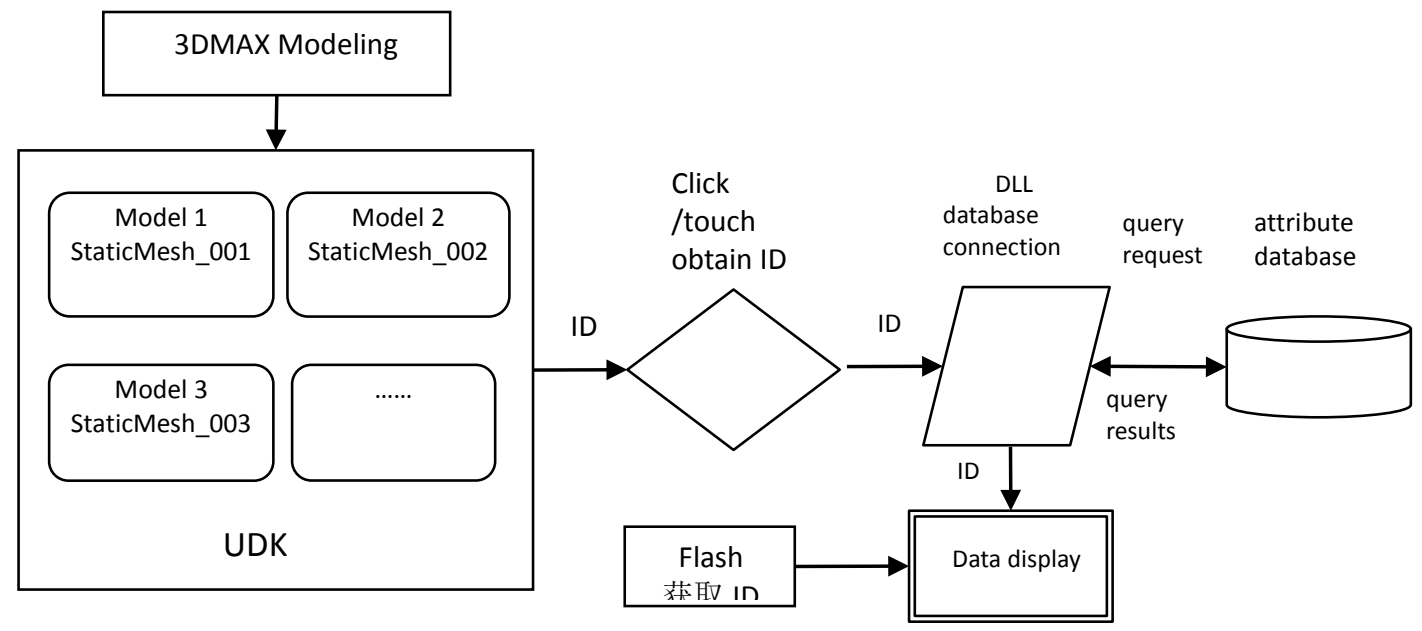

Figure. 3 Data flow chart of display system

\section{TCP/IP Protocol}

First of all, the network communication protocol should be selected for the establishment of computer network, the TCP/IP Protocol is selected in this system.

TCP/IP was originated from a packet switching network research project in the 60's of the last century, which the United States government funded. And it is submerged into BSD UNIX by California State University Berkeley in the 80's. This action promotes the combination of the TCP/IP and UNIX. It greatly promotes the research and development of TCP/IP. The TCP protocol is connection-oriented transmission control protocol, although TCP was a reliable transport mechanism packet reordering affected its performance, TCP provides a mechanism for data validation and data retransmission and guarantees transmitted data is able to reach the opposite party of communication just like the phone.

\section{Data display}

Before building the virtual environment, the relationship between the scene node and the other simulation node needs to be determined according to the requirement of simulation. This relationship is often the relationship between objects. The models, including scenes 3D geometric model and sound, terrain modeling are built according to the relationship. The terrain modeling and the geometric modeling should be divided because the real terrain data and particular terrain modeling software is used in the terrain modeling.

The interactive design of user interface, it affects the display performance of data results. To control the user interface interaction of the main elements in the UDK of HUD, two ways of display 
elements on the screen are taken, one is the Canvas painting, another way is ScaleformGFx video.

(1) Canvas

The Canvas class contains the screen (or some other surface textures, scripts can be described in the model surface). All the required functions are described the text and images. Every time after a painting circle, Canvas allocates a new HUD, and use the Canvas screen painted as necessary elements. The size of the canvas mode operation can be big or small, because all rendering includes window, text, animation switch, all need to write script one point one points, so we will greatly increase the workload.

(2) ScaleformGFx

The ScaleformGFx integrates a set of video playback functions, and it can use the built-in Adobe Flash animation as the game's UI display. It is a separate object contains by a set of video, video player, video of the class. Users can create a video player in the HUD, control Flash complete animation. And through the Flash Action programming Script, some interaction effect can reach Flash. This study adopts the way of query results show UI. Artists of the Flash design a good display effect of animation, animation will be generated as.Swf files, UDK is integrated into the file, generate the built-in video. When the DLL completes the query returns query results, animation is displaying, and query results are taken as the parameters for transferring to the video, then the results can be displayed according to the result of Flash, the simulation result is shown in Figure 4.

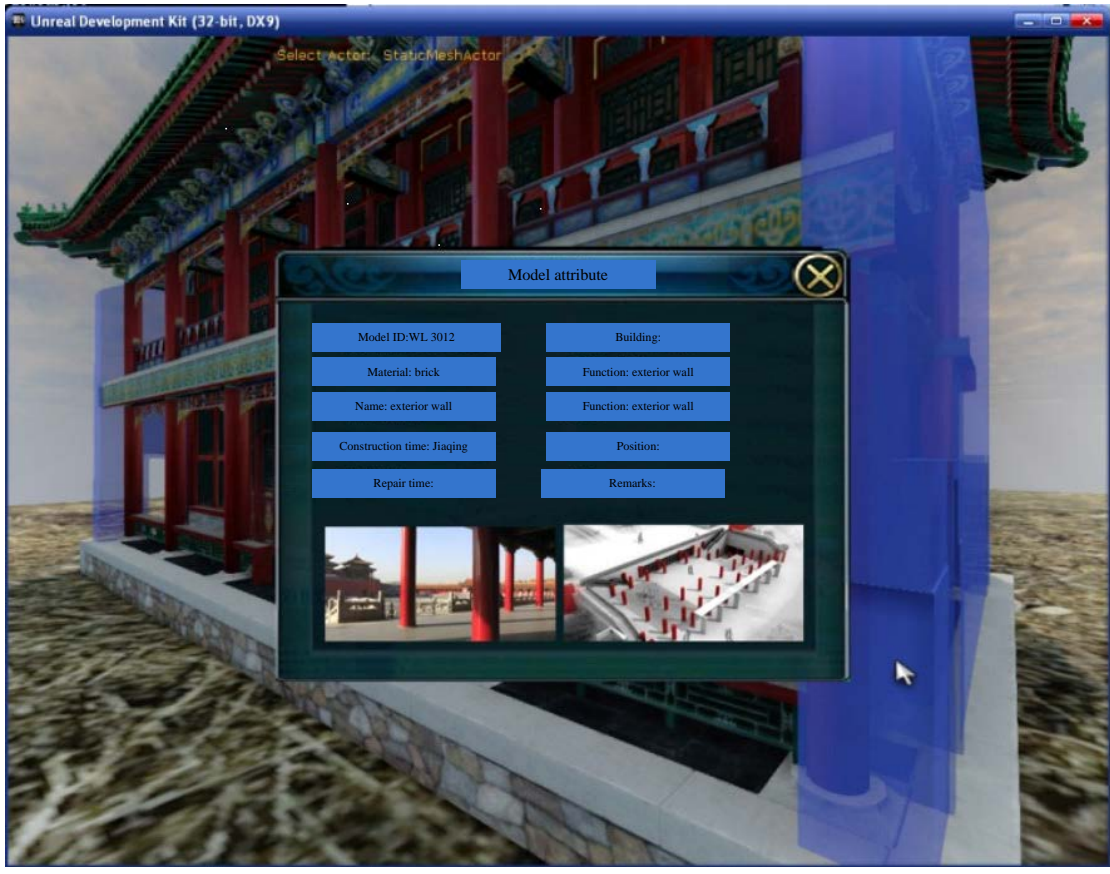

Figure 4 Schematic diagram of attribute data display

\section{Summary}

In this paper, the UDK technology and UnrealScript script programming language are studied, ombined with UDK technology and 3DMAX, Flash, Photoshop and other software, developed the display system of high precision model, an attribute query and display system of ancient buildings are designed. The technical route has a high efficiency and display effect friendly, and it has a good application prospect $\mathrm{n}$ the field of cultural heritage digital display.

\section{Reference}

[1] GE Lizhi. Visual simulation of UUV Attack Model Based on Whole Trajectory Control Analysis[J].Ship Electrolic Engneering, 2015,35(3):137-141.

[2] ZOU Shengyu, LIU Zhen, GAO Haibo etc. Tracking Control Method for Suspended Floater Based on Time Integral of Disturbance Force[J]. ROBOT, 2015,37(1): 1-8,16. 
[3] WANG Lei, LIU Haitao, LIANG Tao, et al. Modeling and Analysis of Dynamic Response of Servo Feed System under Low Frequency Excitation[J]. Journal of Mechanical Engineering, 2015, 51(3): 18-28.

[4] CHENG Guoying, WANG Yan. Multi-mobile Agent collaborative control data separation method in wireless sensor network. Journal of Computer Applications, 2015, 35(4): 910-915.

[5] Hu Yaoyun, in chifeng area topography of $3 \mathrm{~d}$ modeling and its application [J]. Science and technology,2012,28(4):79-81.

[6] Feng Chaorong, based on the dynamic movement particle model of complex three-dimensional fabric simulation technology research [J]. Science and technology,2012,28(10):64-66. 